\title{
The Effect of Variable and Uniform Densities on the Inertial Properties of Cadaver Segments, a Comparison of Frozen and Thawed Densities, and Segment Densities Related to Endomorphy and Ectomorphy
}

\author{
By S. Alan Lephart* \\ John H. Bolte, IV $V^{\dagger}$ \\ Christopher B. Albery
}

This study was designed to answer three questions. Is there a significant difference between the inertial properties of the segments, such as center of mass location and magnitude of principal moments of inertia, when variable density within the segment is compared to a comparable uniform density model? Is there a significant difference between frozen and thawed segment densities? Is there a significant difference in the density of ectomorphic and endomorphic body types? Two cadavers of different body mass indices were segmented into 16 segments each and further cross-sectioned into horizontal slices 1 to $2 \mathrm{~cm}$. thick. Densities of frozen and thawed segments and sections were measured by immersion, and variable and uniform density models were examined regarding inertial properties. A stacked ellipse model having identical geometries for both conditions was used to attempt to answer these questions. Variable and uniform densities yielded results that differed by a maximum of about $0.3 \%$ for all segments and which did not approach significance in either center of mass location or magnitude of principal moments of inertia. It was concluded that models that include variable density (such as DEXA, MRI, CT) increase the expense of estimating inertial properties without any appreciable gain in accuracy over less expensive models based on topography alone. There were significant density differences $(\mathrm{p}<0.01)$ when comparing ectomorphy with endomorphy. It is suggested that it may be useful to consider density differences due to body somatotypes when performing motion analysis. Comparison of frozen density and thawed density showed significant differences in both subjects $(\mathrm{p}<0.01)$, and applied to all segments. It is also suggested that since frozen density is significantly different from thawed density, that thawed density,

\footnotetext{
*Assistant Professor, Fort Lewis College, USA.

${ }^{\dagger}$ Associate Professor, The Ohio State University, USA.

${ }^{\dagger}$ Principal Scientist and Manager of Biodynamics CRADA, Infoscitex Corp. (Wright Patterson Air Force Base, Ohio), USA.
} 
being more comparable to analyses in vivo, should always be used in analysis of movement.

\section{Introduction}

In order to calculate inertial properties of the human body for the purpose of mechanical analysis of human movement skills, most researchers consider the body as a collection of rigid objects that are linked together at the joints, even though the segments of the body are not truly rigid. The segments usually chosen include the long bone segments (upper arms, forearms, thighs and lower legs), the hands, the feet, the head and the torso. The torso can either be considered as a single segment or divided into upper and lower, or upper, middle and lower, segments. The important inertial properties necessary for analysis of skills are the mass, the position of the center of mass (COM) in three dimensions, and the three principal moments of inertia and their direction cosines relative to a fixed axis set (usually the Cardinal axes). Researchers have performed a number of cadaver studies to determine the masses and COM positions of body segments (Braune and Fischer 1889; Clauser, McConville, and Young, 1969; Dempster, 1955). However, the only thorough treatment regarding principal moments of inertia is the work by Chandler and his co-workers (Chandler, McConville, Reynolds, and Young, 1975), which has apparent flaws in the resulting data. For any real object, no one principal moment of inertia can be greater than the sum of the other two. In the heavier segments in Chandler's work, five of the six torsos and seven other segments as well as two of the three total body values in the standing position, violate this rule, which results in the momental ellipsoids representing these segments having imaginary dimensions. These errors appear to be associated with the mass of the segment, which suggests a flaw in the pendulum technique that may make the data unreliable.

A number of researchers have proposed geometric models for the segment inertial properties (Lephart, 1973; Whitsett, 1962; Woolley, 1972). The most notable of these are the widely used model proposed by Hanavan (1964) and the mathematically more complex but less widely used model suggested by Hatze (1979). More sophisticated models that emulate geometrical shapes, such as that developed by Herron, Cuzzi and Hugg (1976) and used by McConville, Churchill, Kaleps, Clauser and Cuzzi (1980) have also been used.

Dual energy X-ray absorptiometry (DEXA) has been used in studies to estimate tissue density and, with other techniques, to estimate inertial properties with varying degrees of success (Durkin, Dowling, and Andrews, 2002; Ganley and Powers, 2004). Pearsall, Reidt and Livingston, (1996) used computer tomography (CT) to the same end, and magnetic resonance imaging (MRI) was used by Cheng, Chen, H., Chen, C.S., Lee and Chen, C.Y. (2000) in a similar study. Most of these studies have 
suggested that density differences within segments are of little consequence in determining inertial parameters.

Surface scanning techniques such as photogrammetry (Jensen, 1978; McConville, et al., 1969) have been used with uniform density models as have other surface scanning techniques, such as infrared (Norton, Donaldson and Dekker, 2002) and laser scanning (Whitestone, personal communication, 2000) to determine segment inertial properties based on topography.

A number of regression models based upon cadaver data and anthropometric measurements have been devised (Barter, 1957; Chandler et al, 1975; Dempster, 1955; McConville et al., 1980; Zatsiorsky, and Seluyanov, 1983).

In most models (other than DEXA, MRI and CT scanning), it is usually assumed that the density is uniform throughout the segment. Whether such an assumption is justified is questionable (Ackland, Henson, and Bailey, 1988). The segments are composed of muscle, connective tissue, fat and bone and, in the case of the torso, internal organs such as lung, liver, intestines, etc. It is known that these different tissues do not possess the same density, nor are they evenly distributed within the segment.

The density of fat is generally reported to be about 0.900 to 0.917 (Brozek, Grande, Anderson, and Keys, 1963; Fidanza, Keyes and Anderson, 1953). Muscle density is about 1.04 to 1.12 and the density of connective tissue, nerve and tendon is approximately 1.05 (Amar, 1920; Nadeshdin, 1932). Bone density varies from about 1.15 to 1.95 , depending on its location in the body (Ross et al., 1986). Of the internal organs, the liver at 1.05 is somewhat denser than brain (1.03) or intestine (1.03), while healthy lung is considerably less dense, being about 0.3 (Gamett et al., 1977). Bone makes up about $25 \%$ of the weight of the body, muscle about $40 \%$, the viscera about $22 \%$ and the remaining skin, fat and other tissue about 13\% (Dempster, 1955; Stouffer, 1968).

Although there is not a vast disparity in density, the distribution of these tissues within any segment is far from uniform. For example, when one considers the skull, it is clear that the bone acts as a protective shell around the brain and it is known that bone is denser than brain matter. Therefore, one might conclude that the principal moments of inertia of the head should be greater than would be the case if the head were to be considered as having uniform density throughout. Similarly, both the thigh and upper arm segments and, to a lesser extent the forearms and lower legs, have a higher proportion of bone at the ends of the segment, with the shafts more or less centrally located along the longitudinal axis. This might suggest larger moments of inertia with regard to the axes perpendicular to the long axis of the bone, but a reduced moment of inertia about the long axis itself. With regard to the torso, the expectation would depend upon whether the torso was further subdivided. Considered as an entire unit it would mimic the long bone segments with a greater proportion of bone in the shoulders and pelvis, and the spine more 
centrally concentrated. The lung area is particularly less dense than the remainder of the torso. Various segmentation plans of the torso, such as the two and three section torso models, may suggest other possible anomalies to the uniform density assumption.

In addition to possible changes in the moments of inertia of the segments due to variable density, such variability can also affect the COM position. The thigh, for example, has a greater concentration of bone at the distal end than at the proximal end and an even lower proportion in the middle of the thigh, and a greater fat content at the proximal end, all of which might place the COM nearer the distal end than if the density were truly uniform.

\section{Statement of the Problem}

The problem is that it is not known how much the variability of density within the segments affects the segments' inertial properties when compared with models assuming uniform density. A second problem is that the difference between frozen and thawed cadaver densities has not been examined in a comparative study. Finally, the differences in segment densities between body types (ectomorphic and endomorphic) have not been thoroughly researched.

\section{Purposes of the Study}

One purpose of this study is to compare the inertial properties estimated using uniform density with those of geometrically identical segments that assume variable density over the length of the segment. Special attention is given to the two principal axes at right angles to the longitudinal axis of the segment (the axis perpendicular to the horizontal plane). A second purpose is to compare the densities of frozen segments to thawed segments for the same cadavers. The final purpose of this study is to determine if significant differences in density exist between ectomorphic and endomorphic somatotypes.

\section{Procedure}

For this study, two male cadavers were selected for examination. Neither had visible atrophy, wasting, emaciation or deformity, and neither appeared to be particularly arthritic. The specimens were selected to represent endomorphy and ectomorphy on the assumption that greater or lesser fat content might affect results. Subject number one was an endomorphic 87- year old, 1.85 meters tall, weighing $112.27 \mathrm{~kg}$., and having a Body Mass Index (BMI) of 32.8. Subject number two was a moderately ectomorphic 94-year old, 1.73 meters tall, weighing $67.27 \mathrm{~kg}$, and having a BMI of 22.7. Both subjects were Caucasian.

Prior to segmentation, both subjects were stored for three days in a full body freezer, the temperature of which was maintained between $-14 \mathrm{C}^{\mathrm{o}}$ and $-16 \mathrm{C}^{\mathrm{o}}$. Each subject was divided into 16 segments. A reciprocating saw was used to separate the limbs from the torso. All other cuts were made 
using a band saw. The segments examined were the head, upper, middle and lower torso, both upper arms, both forearms, both hands, both thighs, both lower legs, and both feet.

Except for the division of the torso into three sub-sections, the planes of segmentation followed the plan used by Chandler et al. (1975). The torso was divided into three segments. The upper torso was separated from the remainder by a horizontal cut (considered in the normal anatomical position) through the tip of the xiphoid process. This allowed for most of the lung and much of the rib cage to remain in the upper torso. The remainder of the torso was separated by a horizontal cut at the level of the iliac crest, placing the entire pelvis in the lower torso and the intestines predominantly in the middle torso.

After the segmentation process, each segment was weighed using a Sartorius precision electronic balance with a capacity of $30 \mathrm{~kg}$ and weighing in increments of 1 gram \pm 1 gram.

The segments were then weighed while immersed in water using an Ohaus electronic balance having a capacity of $10 \mathrm{~kg}$ and a resolution of 0.1 gram \pm 0.1 gram. In cases where segments had densities less than 1.0 , a weight was attached to make the segment negatively buoyant and the effect of the weight was later calculated and removed mathematically. From these measurements, the frozen densities of all segments were determined from the following equation:

Density = Mass in Air / (Mass in Air - Mass in Water), Or, $\delta=\mathrm{m} / \mathrm{v}$, where $\mathrm{m}=$ mass and $\mathrm{v}=$ volume.

While still frozen, the segments were photographed in both the frontal and sagital planes to provide an outline of the segments from those perspectives for later use. The overall length of each segment was also recorded, as was the length between proximal and distal centroids. The process of density determination was then repeated after the sections were allowed to thaw, after which the segments were re-frozen.

Each segment was then sliced into a number of cross-sections. The thickness of the sections varied from segment to segment and, to some extent, within segments, although every effort was made to ensure reasonably consistent thickness of the sections for each segment. Sections varied in thickness from about one centimeter (certain arm and leg segments) to two to three centimeters for the torso and head segments. Once the sections were cut, their densities were determined after which they were allowed to thaw. The densities of the thawed slices were then measured. This was done so that a comparison could be made between frozen and thawed densities.

This resulted in a unique density for each section with regard to its longitudinal position within the segment. As anticipated, those sections high in bone and/or low in fat showed greater density than sections in which the reverse was true. As might be expected, the frozen density was less than the thawed density for each section due to expansion during the 
freezing process, which resulted in a larger volume without a corresponding increase in mass (the 'iceberg' effect).

As is always the case, material was lost during both the segmenting and the sectioning processes. The loss of material in segmenting was between $1 \%$ and $2 \%$. The loss during sectioning was about $12 \%$ to $16 \%$ depending on the segment mass and the number of cross-sections. To make the study more realistic and comparable to the intact cadaver, the masses and volumes of the sections were adjusted proportionally to correct for the loss of material through sectioning. The integrity of the relationship between total thawed segment mass and volume (and thus mean density) was held constant during this procedure.

The next step was to digitize the sagital and frontal outlines of each segment from the photographs taken previously. In these photographs, the longitudinal axis (z-axis) was defined as a line extending from the distal geometric centroid of the cut surface of the segment to the geometric centroid of the cut surface of the proximal end (see Figure 1).

Figure 1. Illustration of an Upper Arm Showing a Typical Elliptic Crosssection

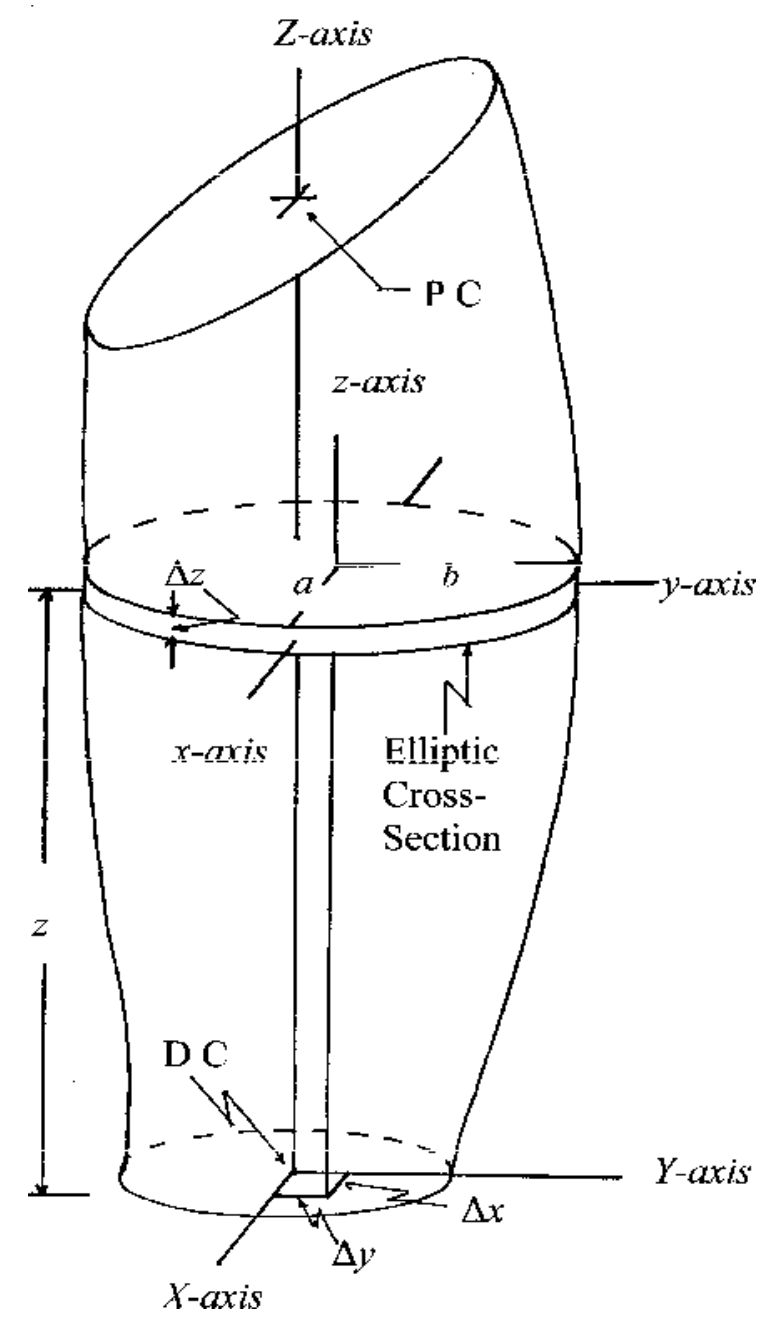


In Figure 1, the segment axes are labeled $X, Y$ and $Z$ and the principal axes of the cross-section are labeled $x, y$ and $z$. The position of the center of volume of the cross-section relative to the segment axes is given by the values $\Delta x, \Delta y$, and $z$. The defining dimensions of the elliptic cross-section are given by $a, b$ and $\Delta z$. PC and D C are the segment proximal and distal centroids, respectively.

The distal and proximal centroids were defined as the geometric centers of the cut surfaces at the distal and proximal ends, respectively. These were assessed by determining the mid-points of the maximum breadth and depth of each cut surface. The $x$-axis was defined as the axis perpendicular to the frontal plane, and positive to the anterior. The $y$-axis was defined as the axis perpendicular to the sagital plane, and positive to the cadaver's left side. The z-axis was defined as perpendicular to the horizontal plane, and positive upward, thus forming the familiar right-hand triad. It should be noted that the cross sectioning of each foot segment took place along the $x$ axis rather than the z-axis, as was the case with all other segments.

The hands, feet and head posed a particular problem since no "cut surface" existed at the vertex of the head, at the distal end of the hand or at either end of the foot. In the case of the head, the z-axis was defined as being perpendicular to the Frankfort plane and intersecting the midpoint of the line joining the right and left tragia (which was defined as the $y$-axis). The $x$-axis was normal to the $y$ - and $z$-axes.

The $x$-axis of the foot was chosen to extend from the center of the heel to the tip of the second toe (as viewed from above) and parallel to the sole of the foot. In the case of the hand, the z-axis was chosen to extend from the tip of the middle finger to the proximal centroid of the cut surface at the wrist. The $y$-axis was chosen as being parallel to the palm of the hand and at a right angle to the z-axis. The axis system of the hands for both subjects was somewhat inconsistent with the Cardinal axis system because of the natural hand position assumed in death.

The photographic outlines allowed determination of the geometric position of each cross-section relative to other cross-sections within the segment as well as to the Cardinal anatomical axes at the distal end of the segment.

To simplify the comparison of the variable and uniform density models, it was assumed that each cross-section was a thin elliptic cylinder in shape (the commonly used "stacked ellipse" model). This resulted in a number of elliptic cross-sections, which facilitated numerical integration of the sections and ultimately allowed determination of the inertial properties for both the variable and uniform density models.

The digitized outlines were used to determine the relative depth and breadth of each cross-section measured at right angles to the longitudinal axis (and in the transverse plane). The outlines also yielded the geometric center of volume position of the cross-section ( $\Delta x$ and $\Delta y$ in the transverse plane relative to the longitudinal axis) and the longitudinal position of the center of volume along the long axis of the segment $(z)$ for each section. 
Next the principal radii ( $a$ and $b$ ) were determined for the many crosssections. These radii were proportionally adjusted to agree with the expected volume of the elliptic cross-sections as determined by the immersion process.

The volume, center of volume and volume moments of inertia of each elliptic cylinder were computationally identical for both the variable density and uniform density models in all segments. The cross-sectional volumes were then determined geometrically.

The center of volume of each section was defined by the coordinates $\Delta x, \Delta y$ and $z$ relative to the Cardinal anatomical axes at the distal end of the segment. This meant that the center of volume of each cross-section could be offset from the longitudinal anatomical axis of the segment by a value of $\Delta x$ and/or $\Delta y$. The magnitude along the z-axis was determined by the longitudinal position of the section relative to the distal end of the segment.

The elliptic cylinder volume moments and products for both models were identical and were computed from the known parameters ( $v, a, b, \Delta x$, $\Delta y, \Delta z$ and $z$ ) for each section geometrically.

For the uniform density model, each cross-section was treated as if it had the mean density of the entire segment. For the variable density model, each cross-section was assigned a density based upon the experimental data according to its longitudinal position within the segment.

Once the volume inertial properties for each section had been determined, which were identical for both the variable and uniform density models, it remained only to determine the inertial properties of the entire segment as a collection of the elliptic cross-sections. These properties included mass, COM position and principal mass moments of inertia about the segment COM as they were related to the anatomical axes of the segment. In the case of the uniform density model, the mass properties of inertia of each cross-section were determined by multiplying the volume properties by the mean density of the segment. Since both first and second mass moments are additive, these values were used to determine the COM position and the inertia tensor elements for the entire segment.

Determining the inertial properties of the variable density model was performed in exactly the same way except that each cross-section included a unique density, based upon the research findings rather than using the mean density for the entire segment. Since the overall masses and volumes (and thus densities) of both models of the segment were identical, any differences in COM location and/or principal moments of inertia were due entirely to the variable density of the individual cross-sectional slices.

Once the COM position was calculated, the tensor elements were transposed to the segment COM using the parallel axis theorems for both moments and products of inertia. Once these elements were determined, the principal moments and direction angles were found by setting the expanded determinant of the inertia tensor equal to zero and calculating the characteristic values of the resulting equation (eigenvalues and eigenvectors). 
It should be remembered that the geometric properties of both the variable and uniform density models in this study were identical. The sole difference between the models was that the density of one model was assumed uniform, while the other varied as a function of longitudinal position of the cross-sections within the segment.

Of course, the use of cadaver material to simulate living subjects comes with the usual caveats. However, even given these restrictions, the relative variability of density within the segments should approximate that of a living subject.

One problem did arise during the segmentation procedure. It was discovered that the ectomorphic specimen had a surgical implant (hip replacement) in his right thigh. This was not apparent during the initial inspection of the body and there was almost no visible scar to indicate that an operation had ever taken place. Since the replacement was made of stainless steel and increased the weight of the thigh, it was decided not to include the data from the subject's right thigh in this study. Instead, it was assumed that the right thigh would not be appreciably different from the left, so the data from the left thigh was duplicated for the right thigh. The pelvis was virtually unaffected by the implant with the exception of a slightly enlarged acetabulum on the right side. It was felt that this would not appreciably affect the inertial properties of the lower torso.

\section{Results}

The results of this research are presented in tabular form. Although it is customary to use kilograms and meters in presenting scientific results, grams and centimeters were used as units of measure in this study to facilitate comparison to the works of Dempster (1955) and Chandler et al. (1975), the two major proponents of cadaver research.

The following abbreviations are used to represent the segments:

$\begin{array}{lll}\text { HED }=\text { Head } & \text { UTO = Upper Torso } & \text { MTO = Middle TorsoR } \\ \text { LTO = Lower Torso } & \text { UA = Right Upper Arm } & \text { LUA = Left Upper } \\ \text { RFA = Right Forearm } & \text { LFA = Left Forearm } & \text { RHA = Right Hand } \\ \text { LHA = Left Hand } & \text { RTH = Right Thigh } & \text { LTH = Left Thigh } \\ \text { RLL = Right Lower Leg } & \text { LLL = Left Lower Leg } \\ \text { RFT = Right Foot } & \text { LFT = Left Foot } & \end{array}$


Vol. 1, No. $2 \quad$ Lephart et al.: Revenues from related Parties Transactions...

Table 1. Cadaver 1 - Masses, Volumes and Densities of Segments

\begin{tabular}{ccrrcr}
\hline \multirow{2}{*}{ Segment } & \multirow{2}{*}{ Mass } & \multicolumn{3}{c}{ Frozen } & \multicolumn{2}{c}{ Thawed } \\
\cline { 2 - 6 } & & Volume & Density & Volume & Density \\
\hline HED & 5,483 & 5,156 & 1.063 & 5,030 & 1.090 \\
UTO & 25,330 & 25,735 & 0.978 & 25,975 & 0.982 \\
MTO & 18,925 & 19,618 & 0.960 & 19,470 & 0.972 \\
LTO & 13,658 & 13,862 & 0.975 & 13,936 & 0.980 \\
RUA & 3,267 & 3,250 & 1.005 & 3,216 & 1.016 \\
LUA & 3,226 & 3,197 & 1.009 & 3,144 & 1.026 \\
RFA & 1,550 & 1,500 & 1.033 & 1,474 & 1.051 \\
LFA & 1,509 & 1,450 & 1.041 & 1,434 & 1.052 \\
RHA & 570 & 540 & 1.057 & 533 & 1.071 \\
LHA & 572 & 538 & 1.063 & 532 & 1.076 \\
RTH & 14,634 & 14,632 & 0.989 & 14,678 & 0.997 \\
LTH & 12,352 & 12,735 & 0.970 & 12,389 & 0.997 \\
RLL & 4,247 & 4,128 & 1.029 & 4,080 & 1.041 \\
LLL & 4,476 & 4,321 & 1.036 & 4,304 & 1.040 \\
RFT & 1,237 & 1,173 & 1.055 & 1,160 & 1.067 \\
LFT & 1,229 & 1,167 & 1.053 & 1,138 & 1.080 \\
\hline
\end{tabular}

Table 2. Cadaver 2 - Masses, Volumes and Densities of Segments

\begin{tabular}{cccccc}
\hline \multirow{2}{*}{ Segment } & \multirow{2}{*}{ Mass } & \multicolumn{2}{c}{ Frozen } & \multicolumn{2}{c}{ Thawed } \\
\cline { 3 - 6 } & & Volume & Density & Volume & Density \\
\hline HED & 4,198 & 4,007 & 1.048 & 3,833 & 1.095 \\
UTO & 17,588 & 18,139 & 0.967 & 18,076 & 0.973 \\
MTO & 13,734 & 13,955 & 0.984 & 13,831 & 0.993 \\
LTO & 8,722 & 9,086 & 0.960 & 8,775 & 0.994 \\
RUA & 1,574 & 1,579 & 1.009 & 1,518 & 1.037 \\
LUA & 1,632 & 1,631 & 1.001 & 1,563 & 1.044 \\
RFA & 688 & 670 & 1.028 & 655 & 1.051 \\
LFA & 728 & 703 & 1.036 & 692 & 1.052 \\
RHA & 377 & 357 & 1.054 & 361 & 1.043 \\
LHA & 329 & 312 & 1.056 & 317 & 1.037 \\
RTH* & 6,394 & 6,379 & 1.002 & 6,293 & 1.016 \\
LTH & 6,394 & 6,379 & 1.002 & 6,293 & 1.016 \\
RLL & 1,727 & 1,674 & 1.031 & 1,651 & 1.046 \\
LLL & 1,549 & 1,488 & 1.030 & 1,475 & 1.050 \\
RFT & 871 & 854 & 1.008 & 850 & 1.025 \\
LFT & 762 & 746 & 1.021 & 731 & 1.042 \\
\hline
\end{tabular}

The units in Tables 1 and 2 are grams for mass and cubic centimeters for volume. Density is, of course, the ratio of the two. 
Table 3 presents the relative proportions (percentage) of the segments to the entire body in terms of mass, volume and length.

Table 3. Segment Percentages of Total Body Mass, Volume and Stature

\begin{tabular}{ccccccc}
\hline \multirow{2}{*}{ Seg. } & \multicolumn{3}{c}{ Cadaver 1 } & \multicolumn{3}{c}{ Cadaver 2 } \\
\cline { 2 - 7 } \% Mass & \% Vol. & \% Stat. & \% Mass & \% Vol. & \% Stat. \\
\hline HED & 4.9 & 4.5 & 13.1 & 6.2 & 5.7 & 13.1 \\
UTO & 22.6 & 23.0 & 20.8 & 26.1 & 27.0 & 20.8 \\
MTO & 16.9 & 17.3 & 12.5 & 20.4 & 20.7 & 12.4 \\
LTO & 12.2 & 12.4 & 16.2 & 13.0 & 13.1 & 16.2 \\
RUA & 2.9 & 2.9 & 19.8 & 2.3 & 2.3 & 19.6 \\
LUA & 2.9 & 2.8 & 19.6 & 2.4 & 2.3 & 19.7 \\
RFA & 1.4 & 1.3 & 16.0 & 1.0 & 1.0 & 15.1 \\
LFA & 1.3 & 1.3 & 16.1 & 1.1 & 1.0 & 15.5 \\
RHA & 0.5 & 0.5 & 10.8 & 0.6 & 0.5 & 10.4 \\
LHA & 0.5 & 0.5 & 11.1 & 0.5 & 0.5 & 9.6 \\
RTH* & 13.0 & 13.1 & 31.3 & 9.5 & 9.4 & 31.3 \\
LTH & 11.0 & 11.0 & 31.2 & 9.5 & 9.4 & 31.3 \\
RLL & 3.8 & 3.6 & 23.2 & 2.6 & 2.5 & 22.6 \\
LLL & 4.0 & 3.8 & 23.5 & 2.3 & 2.2 & 21.4 \\
RFT & 1.1 & 1.0 & 14.1 & 1.3 & 1.3 & 13.9 \\
LFT & 1.1 & 1.0 & 14.7 & 1.1 & 1.1 & 14.1 \\
\hline
\end{tabular}

The units in Table 3 are all percentages of total body mass, total body volume, or stature.

Table 4 shows the segment overall lengths (LOA), the lengths from distal to proximal centroids (LCC), the distance from the proximal centroid of the segment to the COM (LPC) and the percentage of that distance of the centroid-to-centroid length (\% LPC). The head lengths were measured from the vertex, the hands from the wrist joint center to the tip of the middle finger, and the feet from the heel to the tip of the second toe. The foot lengths are measured along the $x$-axis from heel to toe.

Table 4. Segment Length Over-All (LOA), Length from Proximal to Distal Centroid (LCC), and COM Position Relative to the Proximal Centroid (LPC)

\begin{tabular}{ccccccccc}
\hline \multirow{2}{*}{ Seg. } & \multicolumn{4}{c}{ Cadaver 1 } & \multicolumn{5}{c}{ Cadaver 2 } \\
\cline { 2 - 9 } & LOA & LCC & LPC & \% LPC & LOA & LCC & LPC & \%LPC \\
\hline HED & 24.3 & 15.3 & 10.0 & 65.4 & 22.6 & 16.7 & 11.1 & 66.5 \\
UTO & 38.5 & 38.5 & 21.2 & 55.1 & 35.9 & 35.9 & 21.1 & 58.8 \\
MTO & 23.1 & 23.1 & 11.4 & 49.4 & 21.5 & 21.5 & 9.5 & 44.1 \\
LTO & 30.1 & 30.1 & 11.1 & 37.0 & 28.0 & 28.0 & 10.5 & 37.5 \\
RUA & 36.8 & 29.5 & 14.6 & 49.5 & 33.8 & 28.1 & 13.9 & 49.5
\end{tabular}


Vol. 1, No. $2 \quad$ Lephart et al.: Revenues from related Parties Transactions...

\begin{tabular}{lcccccccc} 
LUA & 36.3 & 29.3 & 14.7 & 50.0 & 34.0 & 28.2 & 14.1 & 50.0 \\
RFA & 29.7 & 27.7 & 11.7 & 42.2 & 26.1 & 23.2 & 9.5 & 40.8 \\
LFA & 29.8 & 26.2 & 11.2 & 42.9 & 26.8 & 24.8 & 10.1 & 40.5 \\
RHA & 20.0 & 20.0 & 8.9 & 44.4 & 18.0 & 18.0 & 8.6 & 47.5 \\
LHA & 20.5 & 20.5 & 8.7 & 42.5 & 16.5 & 16.5 & 7.2 & 43.8 \\
RTH & 58.1 & 44.9 & 17.6 & 39.2 & 54.0 & 41.0 & 15.8 & 38.7 \\
LTH & 57.9 & 44.3 & 17.7 & 40.0 & 54.0 & 41.0 & 15.8 & 38.7 \\
RLL & 42.6 & 43.0 & 17.6 & 40.9 & 39.0 & 36.7 & 15.3 & 41.7 \\
LLL & 42.6 & 43.6 & 17.7 & 40.7 & 36.0 & 36.9 & 15.1 & 40.9 \\
RFT & 26.7 & 26.7 & 12.5 & 46.8 & 24.0 & 24.0 & 10.6 & 44.2 \\
LFT & 27.2 & 27.7 & 12.7 & 45.8 & 24.3 & 24.3 & 10.6 & 43.7 \\
\hline
\end{tabular}

The length units of Table 4 are in centimeters.

Tables 5 and 6 present the principle moments of inertia for the uniform and variable density models, respectively. The units for Tables 5 and 6 are gram centimeters ${ }^{2}$.

Table 5. Cadaver 1 - Principal Moments of Inertia at the COM

\begin{tabular}{ccccccc}
\hline \multirow{2}{*}{ Seg. } & \multicolumn{3}{c}{ Variable Density Model } & \multicolumn{3}{c}{ Uniform Density Model } \\
\cline { 2 - 7 } & $\mathrm{I}_{\mathrm{x}}$ & $\mathrm{I}_{\mathrm{y}}$ & $\mathrm{I}_{\mathrm{z}}$ & $\mathrm{I}_{\mathrm{x}}$ & $\mathrm{I}_{\mathrm{y}}$ & $\mathrm{I}_{\mathrm{z}}$ \\
\hline HED & 203,507 & 319,586 & 333,013 & 202,985 & 318,871 & 330,802 \\
UTO & $5,334,321$ & $3,317,102$ & $3,696,001$ & $5,313,706$ & $3,294,702$ & $3,700,587$ \\
MTO & $2,606,143$ & $1,707,512$ & $2,721,150$ & $2,606,386$ & $1,707,778$ & $2,721,117$ \\
LTO & $1,670,479$ & $1,146,653$ & $1,442,533$ & $1,670,842$ & $1,145,837$ & $1,443,889$ \\
RUA & 272,395 & 294,976 & 60,279 & 271,050 & 293,764 & 60,336 \\
LUA & 272,872 & 288,150 & 56,676 & 271,894 & 287,227 & 56,691 \\
RFA & 82,027 & 83,578 & 16,849 & 81,398 & 82,940 & 16,903 \\
LFA & 78,719 & 79,666 & 15,981 & 78,483 & 79,438 & 16,033 \\
RHA & 14,139 & 11,972 & 4,048 & 14,053 & 11,883 & 4,056 \\
LHA & 13,846 & 11,166 & 4,444 & 13,807 & 11,124 & 4,446 \\
RTH* & $2,910,470$ & $3,048,884$ & 759,649 & $2,908,542$ & $3,047,586$ & 759,523 \\
LTH & $2,514,976$ & $2,545,933$ & 583,663 & $2,512,193$ & $2,542,152$ & 583,948 \\
RLL & 551,976 & 561,741 & 80,444 & 550,244 & 559,983 & 80,708 \\
LLL & 592,041 & 602,182 & 88,376 & 591,797 & 601,967 & 88,755 \\
RFT & 11,327 & 52,810 & 53,090 & 11,332 & 52,767 & 53,045 \\
LFT & 10,631 & 53,315 & 54,307 & 10,640 & 53,176 & 54,162 \\
\hline
\end{tabular}


Table 6. Cadaver 2 - Principal Moments of Inertia at the COM

\begin{tabular}{ccccccc}
\hline & \multicolumn{3}{c}{ Variable Density Model } & \multicolumn{3}{c}{ Uniform Density Model } \\
\cline { 2 - 7 } & $\mathrm{I}_{\mathrm{x}}$ & $\mathrm{I}_{\mathrm{y}}$ & $\mathrm{I}_{\mathrm{z}}$ & $\mathrm{I}_{\mathrm{x}}$ & $\mathrm{I}_{\mathrm{y}}$ & $\mathrm{I}_{\mathrm{z}}$ \\
\hline HED & 132,226 & 213,266 & 218,608 & 132,114 & 212,898 & 217,847 \\
UTO & $2,991,811$ & $1,861,162$ & $1,982,498$ & $2,972,283$ & $1,839,646$ & $1,988,179$ \\
MTO & $1,635,530$ & 975,118 & $1,554,260$ & $1,635,046$ & 974,927 & $1,554,277$ \\
LTO & 782,727 & 569,043 & 633,273 & 781,718 & 568,024 & 633,327 \\
RUA & 110,955 & 116,848 & 14,922 & 110,834 & 118,749 & 14,936 \\
LUA & 128,989 & 134,444 & 14,795 & 129,163 & 134,656 & 14,816 \\
RFA & 36,425 & 36,749 & 3,574 & 36,102 & 36,425 & 3,585 \\
LFA & 37,148 & 37,568 & 3,829 & 36,957 & 37,379 & 3,832 \\
RHA & 8,573 & 7,061 & 2,202 & 8,501 & 6,986 & 2,208 \\
LHA & 7,602 & 6,542 & 1,580 & 7,478 & 6,412 & 1,587 \\
RTH* & $1,126,372$ & $1,161,581$ & 165,935 & $1,123,863$ & $1,159,450$ & 166,398 \\
LTH & $1,126,372$ & $1,161,581$ & 165,935 & $1,123,863$ & $1,159,450$ & 166,398 \\
RLL & 224,420 & 227,426 & 14,845 & 223,360 & 226,376 & 14,963 \\
LLL & 172,612 & 172,757 & 12,613 & 172,193 & 172,234 & 12,695 \\
RFT & 6,246 & 31,103 & 31,513 & 6,252 & 31,018 & 31,437 \\
LFT & 4,494 & 27,953 & 28,640 & 4,496 & 27,867 & 28,552 \\
\hline
\end{tabular}

\section{Discussion}

A number of interesting results came from this research. The density of each segment (either frozen or thawed) indicates that density and bone proportion are positively related. Not surprisingly, the densities of the hands, the feet and the head were greater than other segments due to their high bone and low fat content. Additionally, as has been shown in other studies, the density of the limbs was lower toward the proximal end of both the upper and lower limbs (thigh density was less than lower leg density and lower leg density was less than foot density, etc.).

As might be expected, the densities of some, though not all, of the corresponding parts of the more endomorphic subject were lower than his ectomorphic counterpart. Similarly, frozen density was significantly less than thawed density in both subjects. The lowest cross-sectional density in both subjects corresponded to the area where the lung tissue was most prevalent. It is probable that this would have been even more striking had the subjects not been embalmed. In this study, a test of the lung tissue alone resulted in a density of about 0.67 even after the lungs had been squeezed to expel excess fluid. This indicated that the lungs absorbed embalming fluid readily and, as a result, this tissue was more than twice as dense as would be expected in vivo.

The presence of fatty tissue also reduced the density considerably as was witnessed in this study by the fatty pads found in the upper thighs of the endomorphic specimen and the correspondingly low density of those sections. 
By inspection, it may be seen that the density of frozen material is always less than that of thawed material for any given segment. In fact for each cadaver individually and for both collectively the differences are significant at the $99 \%$ level $(\mathrm{p}<0.01)$. Similar differences may be found between the Chandler density data (1975), which were collected from frozen segments in a cold environment, and that of Dempster's study (1955), which was conducted using thawed material. It may also be seen in these studies that those segments with higher concentrations of bone, such as the head, the hands and the feet, were indeed denser, as would be anticipated.

Excluding the segments in which fat is minimal, such as the head, hands and feet, there is a notable difference between the mean densities of the endomorphic and ectomorphic subjects. The overall density of both subjects was less than has been shown in other studies, being 0.974 for the endomorphic subject and 0.990 for his ectomorphic counterpart. The explanation for this may lie in the fact that in this study, no effort was made to expel air from the segments prior to measurement and the subjects were not chosen to simulate air force flying personnel, as was the case in the Chandler and Dempster studies.

The proportion of total body mass of each segment was reasonably consistent with other studies of this nature. There were differences between the subjects, however. Contrary to what might have been expected, the ectomorphic subject had relatively lower proportions of mass in the limbs and higher proportions in the trunk segments than the endomorphic subject.

It is of interest to note that there is little difference in the relative position of the COM of the segments between the two different somatotypes represented in this study.

An examination of the longitudinal positions of the centers of mass in the variable and uniform density models showed only very slight differences. The differences usually occurred in a predictable direction: that is, the concentration of bone at one end of a segment always resulted in the COM being located nearer that end in the variable density model. However, the mean difference was very small, amounting to less than 1 $\mathrm{mm}$ with a maximum value of $2 \mathrm{~mm}$. Clearly, this amount is insignificant in terms of computing the inertial properties of segments.

Similarly, the principal moments were virtually the same for both the variable and uniform density models. The mean absolute error for $\mathrm{I}_{\mathrm{x}}$ was $0.44 \% \pm 0.37 \%$. For $\mathrm{I}_{\mathrm{y}}$, the mean absolute error was $0.48 \% \pm 0.42 \%$. The differences between the models for $I_{z}$ (principal moment about the longitudinal axis) were much smaller since the mathematical model did not actually consider variable density with regard to this axis. The mean absolute error for $\mathrm{I}_{\mathrm{z}}$ was $0.20 \% \pm 0.24 \%$.

It is difficult to compare the larger subject of this study with subjects from similar studies because of his size. The Dempster and Chandler studies were performed with subjects in a range from about 50 to $90 \mathrm{~kg}$, 
and the larger subject of this study weighed over $112 \mathrm{~kg}$. The fact that the moments of inertia increase exponentially with increases in mass and dimensions is evident when examining the principal moments of inertia of this subject. The smaller subject, on the other hand, is roughly comparable to the mean values of Chandler et al. (1975, pp. 68-96) and weighs about midway between the subjects numbered 15168 and 15250 in Dempster's work (1955). Comparisons made with these subjects from previous studies show similar inertial property values.

\section{Summary and Conclusions}

This study was designed to determine whether variable density along the long axis of body segments would significantly affect the determination of segmental inertial properties. Two cadavers were segmented and further cut into cross-sections to determine the density at various levels along the segment longitudinal axes. A mathematical model was designed to determine the inertial properties of the segments including the position of the COM and the principal moments of inertia. After reviewing the data it was determined that, the inclusion of variable density would not alter the inertial properties to any significant degree. Therefore, it is recommended that models that produce good geometric representations of the topography of segments (biostereometric photogrammetry, laser scanning, etc) be used with known mean segment densities from cadavers in determining segmental inertial properties.

It was also concluded that thawed density is greater than frozen density and since thawed density is more realistic, it should be used when applying findings to subjects in vivo.

There are significant differences in density of ectomorphic and endomorphic body somatotypes. Further study should be undertaken to provide more information regarding the possibility of varying densities based upon body build.

\section{Reference List}

Ackland, T., Henson, P. and Bailey, D. (1988). The uniform density assumption: its effect upon the estimation of body segment inertial parameters. International Journal of Sports Biomechanics 4, pp.146-155.

Amar, J. (1920). The Human Motor (Condensed). AMRL-TDR-63-123, , WrightPatterson Air Force Base, Dayton, OH, p. 163.

Barter, J.T., (1957). Estimation of the Mass of Body Segments. WADC Technical Report 57-260, Astia Document No. 118222, Wright-Patterson Air Force Base, Dayton, $\mathrm{OH}$.

Braune, W. \& Fischer, O. (1889). The Center of Gravity of the Human Body as Related to the Equipment of the German Infantry (Condensed English translation). AMRL-TDR63-123,.Wright-Patterson Air Force Base, Dayton, OH. pp.1-57. 
Brozek, J., Grande, F., Anderson, T. \& Keys, A. (1963). Densiometric analysis of body composition: revisions of some quantitative assumptions. Annals of the New York Academy of Science, (110), pp.113-140.

Chandler, R.F., Clauser, C.E., McConville, J.T., Reynolds, H.M. \& Young, J.W. (1975). Investigation of Inertial Properties of the Human Body. AMRL-TR74-137, Wright-Patterson Air Force Base, Dayton, OH, pp. 61-100.

Cheng, C.K., Chen, H.H., Chen, S.C., Lee, C.L. and Chen, C.Y. (2000) Segment inertial properties of Chinese adults determined from magnetic resonance imaging. Clinical Biomechanics 16: 6 59-566

Clauser, Charles.E., McConville, John.T., \& Young, Joseph.W. (1969). Weight, Volume and Center of Mass of Segments of the Human Body. AMRL-TR-6970 Wright-Patterson Air Force Base, Dayton, OH, pp. 17-62

Dempster, Wilfred. T., (1955). Space Requirements of the Seated Operator. WADC-TR-55-159, Wright Air Development Center, Dayton, OH, Chapter VII.

Durkin, J., Dowling, J. and Andrews, D. (2002) The measurement of body segment inertial parameters using dual energy X-ray absorptiometry. Journal of Biomechanics 35 (2002), pp. 1575-1580.

Fidanza, F., Keys, A. \& Anderson, T., (1953), The density of body fat in man and other mammals. Journal of Applied Physiology, (6), pp. 252-256.

Gamett, E.S., Weber, C.E., Coates, G., Cockshott, W.P., Nahmias, C. \&Lassen, N. (1977). Lung density: clinical method for quantitation of pulmonary congestion and edema. Canadian Medical Association Journal, 116 (2), pp. 153-54.

Ganley, K. and Powers, C. (2004) Determination of lower extremity anthropometric parameters using dual energy X-ray absorptiometry: the influence on net joint momentsduring gait. Clinical Biomechanics 19 (2004), pp.50-56.

Hanavan, E.P., Jr. (1964). A Mathematical Model of the Human Body. AMRL-TR64-102, Wright-Patterson Air Force Base, Dayton, OH, pp. 7-26.

Hatze, H. (1979). A Model for Computational determination of Parameter Values of Anthropomorphic Segments. TWISK-TR-79, Pretoria, South Africa, pp. 2 $1-58$.

Herron, R.E., Cuzzi, J.R. \& Hugg, J. (1976). Mass Distribution of the Human Bodyu Using Biostereometrics. AMRL-TR-75-18, Wright-Patterson Air Force Base, Dayton, OH., pp. 4-52.

Jensen, R. (1978). Estimation of the biomechanical properties of three body types using a photogrammetric method. Journal of Biomechanics 11, pp. 349-358.

Lephart, S.A. (1973). Moments of inertia of human body segments. The Australian Journal of Physical Education, (61), Melbourne, Australia, pp. 5-13.

McConville, J.T., Churchill, T., Kaleps, I., Clauser, C.E. \& Cuzzi, J. (1980). Anthropometric Relationships of Body and Body Segment Moments of Inertia. AFAMRL-TR-80-1 19, Wright-Patterson Air Force Base, Dayton, OH.

Nadeshdin, W.A. (1932). Zur Untersuchung der Miunderwerkigkeit der Organe an Leichen. Duetscge, Z. ges. Gerlichtl. Med., (18), pp. 426-431. (As translated by Sheppard, R.B. in Body Composition in Biological Anthropology. Cambridge, U.K.)

Norton, J., Donaldson, N. and Dekker, L (2002) 3D whole body scanning to determine mass properties of legs. Journal of Biomechanics 35 (2002), pp. 8186. 
Pearsall, D., Reidt, J. and Livingston, L (1996) Segmental inertial properties of the human trunk as determined from computed tomography. Annals of Biomechanical Engineering, Vol. 24, pp. 198-210.

Ross, W.D., Eiben, O.G., Ward, R., Martin, A.D., Drinkwater, D.T. \& Clarys, J.P. (1986). Alternatives for the conventional methods of human body composition and physique assessment. Perspectives in Kinanthropometry, ed. Day, J.A.P., Champaign, IL, pp. 203-220.

Stouffer, J.R. (1968). Ultrasonic Determination of Body Composition. AMRL-TR68-6 1, Wright-Patterson Air Force Base, Dayton, OH, pp. 1-13.

Whitestone, J. (2000). Personal communication. Biodynamics Research Unit, Wright-Patterson Air Force Base, Dayton, OH.

Whitsett, C.E. (1962). Some Dynamic Response Characteristics of Weightless Man. AMRL-TR-63-18, Wright-Patterson Air Force Base, Dayton, OH, pp. 412.

Woolley, C.T. (1972). Segment masses, centers of mass and local moments of inertia for an anthropometric model of man. (as published in Conway, B.A.), Development of Skylab Experiment T-013: Crew/Vehicle Disturbances, Appendix A. NASA TN D-6584.Langley Air Force Base, Hampton,VA.

Zatsiorsky, V. and Seluyanov, V. (1983) The mass and inertia characteristics of the main segments of the human body by means of the best predictive regression equations.(as cited by Matsui and Kobayashi)Biomechanics VIII$B$ pp. 1152-1159 . Champaign:Human Kinetics. 
